\title{
Coarse-graining intramolecular hydrodynamic interaction in dilute solutions of flexible polymers
}

\author{
R. Prabhakar* and E. M. Sevick \\ Research School of Chemistry, Australian National University, Canberra, ACT-0200, Australia \\ D. R. M. Williams \\ Research School of Physical Sciences and Engineering, Australian National University, Canberra, ACT-0200, Australia
}

(Received 19 March 2007; published 31 July 2007)

\begin{abstract}
We present a scheme for coarse-graining hydrodynamic interactions in an isolated flexible homopolymer molecule in solution. In contrast to the conventional bead-spring model that employs spherical beads of fixed radii to represent the hydrodynamic characteristics of coarse-grained segments, we show that our procedure leads naturally to a discrete model of a polymer molecule as a chain of orientable and stretchable Gaussian blobs. This model accounts for both intrablob and interblob hydrodynamic interactions, which depend on the instantaneous shapes of the blobs. In Brownian dynamics simulations of initially stretched chains relaxing under quiescent conditions, the transient evolution of the mean-square end-to-end distance and first normal stress difference obtained with the Gaussian-blob model are found to be less sensitive to the degree of coarse graining, in comparison with the conventional bead-spring model with Rotne-Prager-Yamakawa hydrodynamic interactions.
\end{abstract}

DOI: 10.1103/PhysRevE.76.011809

PACS number(s): $61.25 . \mathrm{Hq}, 83.80 . \mathrm{Rs}, 83.10 . \mathrm{Mj}$

\section{INTRODUCTION}

A long, flexible polymer molecule typically consists of thousands of monomeric units, and detailed atomistic simulations of such a molecule and its surrounding solvent molecules are not practical for those interested in predicting the behavior of dilute polymer solutions at large time scales. However, for the purpose of modeling those dynamical features of a solution that depend on the cooperative motion of the polymer molecule as a whole, it is usually sufficient to represent an isolated polymer chain as a continuous space curve. In this basic structural representation of the polymer, the hydrodynamic characteristics of the space curve of contour length $L$ are modeled by giving it a hydrodynamic thickness of $2 a_{0}$ ("level 0 " in Fig. 1). It is further necessary to incorporate hydrodynamic interactions (HI) between all the points on the polymer contour to accurately predict the dynamical behavior of dilute polymer solutions. Although the continuous space-curve representation of flexible polymers is widely used in analytical theories, computer simulations demand some form of discretization. A first level of discretization consists of representing the chain as a "pearl-necklace" model consisting of a line distribution of touching beads, each of radius $a_{0}$ ("level I" in Fig. 1). Since $a_{0}$ is typically much smaller than the contour length for long polymer molecules and the number of beads required in this representation is large, it is often desirable from a computational point of view to coarse grain further.

The basic motivation behind such coarse graining is one of efficiency: we wish to study the dynamics of the system in terms of a fewer number of variables characterizing the coarser description, without having to first numerically resolve the motion of the chain at the fine-scale description. The formal procedure of coarse graining uses projection op-

\footnotetext{
*Corresponding author; prabhakar@rsc.anu.edu.au
}

erators to eliminate fast degrees of freedom and obtain an equation for the probability distribution of slower variables in the system $[1,2]$. The final Fokker-Planck equation obtained in this formal approach contains complicated memory integrals that are difficult to implement directly in simulations.

In contrast, coarse graining in the popular bead-spring chain model of dilute polymer solutions [3] is achieved through postulation of a completely new structural model, and hydrodynamic interactions between spherical beads in the chain are introduced "by hand." This creates two problems [4-6]. First, the radius of the beads in this model is a new parameter, and its relation to the more fundamental parameter $a_{0}$ is a priori unknown. In fact, this connection is typically not made explicitly, and the bead radius is used as a tunable parameter whose value is chosen carefully such that the bead-spring model reproduces observed dynamical behavior under certain conditions, for instance, in the linearresponse regime. The second problem is that spherically isotropic beads do not accurately model the hydrodynamic characteristics of the large molecular subchains they represent in situations where macromolecules are likely to take up highly stretched configurations. In such conditions, it is found that a large number of beads are required to ensure convergence of predictions with respect to the degree of coarse graining.

The primary objective of the present work is to lay down the theoretical framework for an approach that strikes out an alternative middle path between the extremes of the formal method based on projection operators, and the postulational approach of the standard bead-spring model. We derive equations for a coarser description starting from the FokkerPlanck equation (FPE) for the finer pearl-necklace representation ("level I" in Fig. 1) of the polymer molecule. We thus achieve a more refined treatment of HI than in the conventional bead-spring model by accounting for local structural anisotropy in the molecule, but without introducing new free parameters. On the other hand, we use a series of 


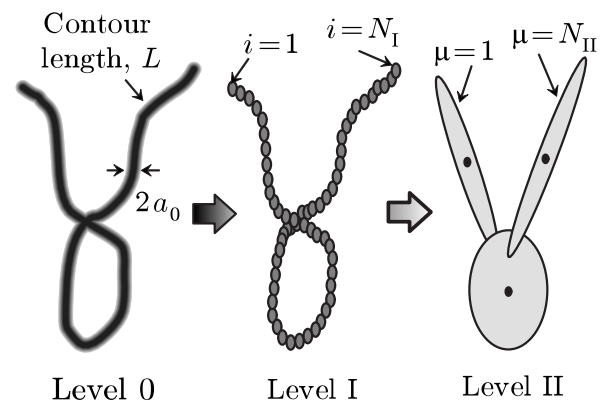

FIG. 1. Coarse graining a continuous chain into a discrete chain of Gaussian blobs. A basic representation of the polymer molecule is that of a continuous space curve (level 0). A first level of discretization leads to the "pearl-necklace" model (level I), which can be further coarse grained as a chain of soft "blobs" (level II).

approximations - some inspired by techniques commonly used in self-consistent mean-field theories for complex fluids, and others guided by intuition - to arrive at a final set of equations that are relatively straightforward to implement in the Brownian dynamics (BD) simulations than those obtained with projection operator methods.

A detailed derivation of the equations of the new coarsegrained model is presented in the following section. The implementation of this model in BD simulations is summarized in Sec. III, and a preliminary comparison of the new model with the conventional bead-spring model is made in Sec. IV.

\section{COARSE-GRAINED FOKKER-PLANCK EQUATION}

As mentioned earlier, a basic structural representation of an isolated linear homopolymer molecule in solution is that of a space curve of contour length $L$ suspended in a continuum Newtonian solvent characterized by its temperature $T$, and its shear viscosity $\eta_{s}$. We do not consider in this study excluded volume effects and restrict our attention to phantom chains. In a dilute polymer solution at equilibrium, the meansquared end-to-end distance $R_{e \text {,eq }}^{2}$ of isolated long, flexible phantom chains is proportional to $L$, and the ratio $b_{K}$ $\equiv R_{e, \text { eq }}^{2} / L$ is defined as the length of a single Kuhn segment in the chain. We are interested here in modeling highly flexible chains in which the number of Kuhn segments $N_{K}$ $\equiv L^{2} / R_{e, \text { eq }}^{2}$ is very large.

At the first level of discretization as a line distribution of beads of radius $a_{0}$-which we will henceforth refer to as the "level I" representation (Fig. 1) - the number of discrete elements in the model is $N_{\mathrm{I}}=L /\left(2 a_{0}\right)$. A single configuration of the polymer chain is completely specified at the level I description by the set of position vectors of the bead centers, $\mathcal{C}^{\mathrm{I}}=\left\{\mathbf{r}_{i} \mid i=1, \ldots, N_{\mathrm{I}}\right\}$. At the "level II" representation of the polymer molecule (Fig. 1), we coarse grain the polymer by partitioning the contour length into $N_{\text {II }}<N_{\text {I }}$ subchains. The $\mu$ th subchain, $\mu=1, \ldots, N_{\mathrm{II}}$, has a contour length $L_{\mu}$, such that $\sum_{\mu=1}^{N_{\text {II }}} L_{\mu}=L$. We do not, for the present, constrain ourselves to partitioning into subchains of equal contour lengths. The $\mu$ th subchain thus contains $n_{\mu}=L_{\mu} /\left(2 a_{0}\right)$ of level I beads. As a matter of convention, we will use italic $(i, j$, etc.) and greek ( $\mu . \nu$, etc.) subscripts to identify level I and level II quantities, respectively. In the level II description, local degrees of freedom are eliminated, and the chain configuration is specified by the set of position vectors of the centers of masses of the subchains, $\mathcal{C}^{\mathrm{II}}=\left\{\mathbf{r}_{\mu} \mid \mu=1, \ldots, N_{\mathrm{III}}\right\}$.

The starting point for our analysis is the FPE for the probability distribution function for the level I configuration, $\psi^{\mathrm{I}}\left(\mathcal{C}^{\mathrm{I}}, t\right)$. For the sake of notational simplicity, we will henceforth not explicitly mention the time dependence of functions. All our knowledge of level I dynamics is contained in the FPE for $\psi^{\mathrm{I}}\left(\mathcal{C}^{\mathrm{I}}\right)$, which we take as given. From this level I FPE, we wish to generate the FPE for the level II configurational probability distribution, $\psi^{\mathrm{II}}\left(\mathcal{C}^{\mathrm{II}}\right)$. The level II FPE thus obtained for $\psi^{\mathrm{II}}\left(\mathcal{C}^{\mathrm{II}}\right)$ must only involve other known functions of the variables $\mathcal{C}^{\text {II }}$. Moreover, the evaluation of these functions should not require any prior knowledge of the level I distribution $\psi^{\mathrm{I}}$. Ideally, deriving the level II FPE should require no further modeling assumptions. If we must make simplifying assumptions, care should be taken not to introduce any new parameters.

We first recall the basic steps in deriving the FokkerPlanck equation for a phenomenological model at the level I representation of the system [3]. It must be recognized that even at this fundamental level, we are already working with a coarse-grained model, since we have replaced all the solvent molecules with a continuum, and have also eliminated all the momentum coordinates of the level I beads. The equation of continuity in level I configuration space for $\psi^{\mathrm{I}}$ is

$$
\frac{\partial \psi^{\mathrm{I}}}{\partial t}=-\sum_{i=1}^{N_{\mathrm{I}}} \frac{\partial}{\partial \mathbf{r}_{i}} \cdot\left(\mathbf{v}_{i} \psi^{\mathrm{I}}\right)
$$

where $\mathbf{v}_{i}$ is the momentum-space averaged velocity of the $i$ th bead. To express the velocity $\mathbf{v}_{i}$ in terms of $\mathbf{r}_{i}$, we invoke the "Stokes-law empiricism" [3], which relates the hydrodynamic frictional drag force $\mathbf{F}_{i}^{h}$ exerted on the polymer molecule at $\mathbf{r}_{i}$ by the Newtonian solvent, to $\mathbf{v}_{i}$,

$$
\mathbf{F}_{i}^{h}=-\zeta_{0}\left(\mathbf{v}_{i}-\boldsymbol{\kappa} \cdot \mathbf{r}_{i}+\sum_{j=1}^{N_{\mathrm{I}}} \boldsymbol{\Omega}\left(\mathbf{r}_{i}, \mathbf{r}_{j}\right) \cdot \mathbf{F}_{j}^{h}\right)
$$

Here, the Newtonian solvent velocity field $\mathbf{v}_{s}(\mathbf{r})$ has been assumed to be homogeneous, that is, $\mathbf{v}_{s}(\mathbf{r})=\mathbf{v}_{0}+\boldsymbol{\kappa} \cdot \mathbf{r}$, where $\boldsymbol{\kappa}=\left(\nabla \mathbf{v}_{S}\right)^{\dagger}$ is the transpose of the velocity gradient, and $\mathbf{v}_{0}$ is the solvent velocity at the origin, which we take to be zero without loss of generality. The friction coefficient of any level I bead is $\zeta_{0}=6 \pi a_{0} \eta_{s}$, and $\boldsymbol{\Omega}\left(\mathbf{r}_{i}, \mathbf{r}_{j}\right)$ is the tensor describing the hydrodynamic interaction (HI) between the beads at $\mathbf{r}_{i}$ and $\mathbf{r}_{j}$. At this stage, we leave the form of the HI tensor $\boldsymbol{\Omega}\left(\mathbf{r}_{i}, \mathbf{r}_{j}\right)$ unspecified, except to note that if we wish to neglect HI completely in the level I model, then $\boldsymbol{\Omega}\left(\mathbf{r}_{i}, \mathbf{r}_{j}\right)$ $=0$ for all $i$ and $j$.

Equation (2) above is rearranged as

$$
\mathbf{v}_{i}=\boldsymbol{\kappa} \cdot \mathbf{r}_{i}-\sum_{j=1}^{N_{\mathrm{I}}} \mathbf{D}_{i j} \cdot \mathbf{F}_{j}^{h},
$$

where 


$$
\mathbf{D}_{i j} \equiv \frac{1}{\zeta_{0}} \boldsymbol{\delta}+\boldsymbol{\Omega}\left(\mathbf{r}_{i}, \mathbf{r}_{j}\right)
$$

is a diffusion tensor, and forms the $(i j)$ th block of $\mathbf{D}$, the $3 N_{\text {I }} \times 3 N_{\text {I }}$ mobility matrix for the level I model. The hydrodynamic drag forces $\mathbf{F}_{i}^{h}$ are eliminated from the equations above by invoking the force balance,

$$
\mathbf{F}_{i}^{h}+\mathbf{F}_{i}^{B}+\mathbf{F}_{i}^{U}=\mathbf{0}
$$

which expresses the fact that inertia is negligible at the length scales under consideration. The Brownian force on the polymer chain at $\mathbf{r}_{i}$ is

$$
\mathbf{F}_{i}^{B}=-k_{B} T \frac{\partial}{\partial \mathbf{r}_{i}} \ln \psi^{\mathrm{I}},
$$

where $k_{B}$ is the Boltzmann constant. The intramolecular force $\mathbf{F}_{i}^{U}\left(\mathcal{C}^{\mathrm{I}}\right)=-\partial U\left(\mathcal{C}^{\mathrm{I}}\right) / \partial \mathbf{r}_{i}$ is a function of the chain configuration and is the resultant force at $\mathbf{r}_{i}$ due to conservative interactions described by the intramolecular potential $U$. As already mentioned, we ignore excluded volume interactions in this study, and $\mathbf{F}_{i}^{U}$ is the resultant of chain tensions responsible for ensuring the connectivity of the polymer molecule and keeping its total length constant at $L$, while maintaining its flexibility such that its equilibrium mean-squared end-toend distance is $R_{e \text { eq }}^{2}$. Using Eqs. (3) and (5) to eliminate $\mathbf{v}_{i}$ and $\mathbf{F}_{i}^{h}$, and using Eq. (6) for the Brownian force, the FokkerPlanck equation governing the time evolution of $\psi^{\mathrm{I}}$ is obtained from the continuity equation (1) as

$$
\begin{aligned}
\frac{\partial \psi^{\mathrm{I}}}{\partial t}= & -\sum_{i=1}^{N_{\mathrm{I}}} \frac{\partial}{\partial \mathbf{r}_{i}} \cdot\left(\boldsymbol{\kappa} \cdot \mathbf{r}_{i}+\sum_{j=1}^{N_{\mathrm{I}}} \mathbf{D}_{i j} \cdot \mathbf{F}_{j}^{U}\right) \psi^{\mathrm{I}} \\
& +k_{B} T \sum_{i, j=1}^{N_{\mathrm{I}}} \frac{\partial}{\partial \mathbf{r}_{i}} \cdot \mathbf{D}_{i j} \cdot \frac{\partial \psi^{\mathrm{I}}}{\partial \mathbf{r}_{j}} .
\end{aligned}
$$

To derive the Fokker-Planck equation for the coarsegrained level II description of the system, we next define the phase function,

$$
\Phi\left(\mathcal{C}^{\mathrm{I}} ; \mathcal{C}^{\mathrm{II}}\right)=\prod_{\mu=1}^{N_{\mathrm{II}}} \delta\left(\mathbf{c}_{\mu}-\mathbf{r}_{\mu}\right)
$$

where $\mathbf{c}_{\mu}=\left(1 / n_{\mu}\right) \sum_{i}^{n_{\mu}=1} \mathbf{r}_{i}$, is the location of the center of mass of the $\mu$ th subchain when the molecule has a level I configuration specified by $\mathcal{C}^{\mathrm{I}}$. It may be noted that we will switch between "local" indices $i_{\mu}=1, \ldots, n_{\mu}$ and "global" indices $i=1, \ldots, N_{\mathrm{I}}$ whenever necessary. The distribution function $\psi^{\mathrm{II}}\left(\mathcal{C}^{\mathrm{II}}\right)$ is thus obtained as a contracted distribution,

$$
\psi^{\mathrm{II}}\left(\mathcal{C}^{\mathrm{II}}\right)=\int \Phi\left(\mathcal{C}^{\mathrm{I}} ; \mathcal{C}^{\mathrm{II}}\right) \psi^{\mathrm{I}}\left(\mathcal{C}^{\mathrm{I}}\right) d \mathcal{C}^{\mathrm{I}}
$$

We can therefore define the conditional probability $\psi^{\mathrm{I} \mid \mathrm{II}}\left(\mathcal{C}^{\mathrm{I}} \mid \mathcal{C}^{\mathrm{II}}\right)$ that the level I configuration of the chain is $\mathcal{C}^{\mathrm{I}}$, given that the centers of masses of the subchains are located at the set of vectors specified by $\mathcal{C}^{\mathrm{II}}$ :

$$
\psi^{\mathrm{I} \mid \mathrm{II}}\left(\mathcal{C}^{\mathrm{I}} \mid \mathcal{C}^{\mathrm{II}}\right) d \mathcal{C}^{\mathrm{I} \mid \mathrm{II}}=\frac{\psi^{\mathrm{I}}\left(\mathcal{C}^{\mathrm{I}}\right) \Phi\left(\mathcal{C}^{\mathrm{I}} ; \mathcal{C}^{\mathrm{II}}\right) d \mathcal{C}^{\mathrm{I}}}{\psi^{\mathrm{II}}\left(\mathcal{C}^{\mathrm{II}}\right)},
$$

with " $d \mathcal{C}^{\mathrm{I} \mid \mathrm{II}}$ " indicating that the probability measure $\psi^{\mathrm{I} \mid \mathrm{II}}$ is defined on the submanifold in level I configuration space on which $\mathbf{c}_{\mu}\left(\mathcal{C}^{\mathrm{I}}\right)=\mathbf{r}_{\mu}, \mu=1, \ldots, N_{\mathrm{II}}$. We will henceforth refer to this submanifold as "the submanifold $\mathbf{c}_{\mu}=\mathbf{r}_{\mu}$." The distributions $\psi^{\mathrm{II}}$ and $\psi^{\mathrm{I} \mid \mathrm{II}}$ satisfy the normalization conditions, $\int \psi^{\mathrm{II}} d \mathcal{C}^{\mathrm{II}}=1$ and $\int \psi^{\mathrm{I} \mid \mathrm{II}} d \mathcal{C}^{\mathrm{I} \mid \mathrm{II}}=1$.

For any function $f\left(\mathcal{C}^{\mathrm{I}}\right)$ defined on the level I configuration space, the average of $f$ is $\langle f\rangle=\int f\left(\mathcal{C}^{\mathrm{I}}\right) \psi^{\mathrm{I}}\left(\mathcal{C}^{\mathrm{I}}\right) d \mathcal{C}^{\mathrm{I}}$, while the average of any function of level II variables, $g\left(\mathcal{C}^{\mathrm{II}}\right)$, is $\langle g\rangle$ $=\int g\left(\mathcal{C}^{\mathrm{II}}\right) \psi^{\mathrm{II}}\left(\mathcal{C}^{\mathrm{II}}\right) d \mathcal{C}^{\mathrm{II}}$. The constrained mean of $f$ over the submanifold $\mathbf{c}_{\mu}=\mathbf{r}_{\mu}$ is

$$
\begin{aligned}
\bar{f} & =\int f\left(\mathcal{C}^{\mathrm{I}}\right) \psi^{\mathrm{I} \mid \mathrm{II}}\left(\mathcal{C}^{\mathrm{I}} \mid \mathcal{C}^{\mathrm{II}}\right) d \mathcal{C}^{\mathrm{I} \mid \mathrm{II}} \\
& =\frac{1}{\psi^{\mathrm{II}}\left(\mathcal{C}^{\mathrm{II}}\right)} \int f\left(\mathcal{C}^{\mathrm{I}}\right) \Phi\left(\mathcal{C}^{\mathrm{I}} ; \mathcal{C}^{\mathrm{II}}\right) \psi^{\mathrm{I}}\left(\mathcal{C}^{\mathrm{I}}\right) d \mathcal{C}^{\mathrm{I}} .
\end{aligned}
$$

For instance, the constrained mean position of the center of mass of the $\mu$ th subchain is obtained trivially as

$$
\begin{aligned}
\overline{\boldsymbol{c}}_{\mu} & =\int \mathbf{c}_{\mu}\left(\mathcal{C}^{\mathrm{I}}\right) \psi^{\mathrm{I} \mid \mathrm{II}}\left(\mathcal{C}^{\mathrm{I}} \mid \mathcal{C}^{\mathrm{II}}\right) d \mathcal{C}^{\mathrm{I} \mid \mathrm{II}} \\
& =\frac{1}{\psi^{\mathrm{II}}\left(\mathcal{C}^{\mathrm{II}}\right)} \int \mathbf{c}_{\mu}\left(\mathcal{C}^{\mathrm{I}}\right) \Phi\left(\mathcal{C}^{\mathrm{I}} ; \mathcal{C}^{\mathrm{II}}\right) \psi^{\mathrm{I}}\left(\mathcal{C}^{\mathrm{I}}\right) d \mathcal{C}^{\mathrm{I}}=\mathbf{r}_{\mu} .
\end{aligned}
$$

Similarly, the constrained mean momentum-space averaged velocity of the $\mu$ th subchain is

$$
\begin{aligned}
\overline{\mathbf{v}}_{\mu} & =\int\left(\frac{1}{n_{\mu}} \sum_{i_{\mu}=1}^{n_{\mu}} \mathbf{v}_{i_{\mu}}\left(\mathcal{C}^{\mathrm{I}}\right)\right) \psi^{\mathrm{I} \mid \mathrm{II}}\left(\mathcal{C}^{\mathrm{I}} \mid \mathcal{C}^{\mathrm{II}}\right) d \mathcal{C}^{\mathrm{I} \mid \mathrm{II}} \\
& =\frac{1}{\psi^{\mathrm{II}}\left(\mathcal{C}^{\mathrm{II}}\right)} \int\left(\frac{1}{n_{\mu}} \sum_{i_{\mu}=1}^{n_{\mu}} \mathbf{v}_{i_{\mu}}\left(\mathcal{C}^{\mathrm{I}}\right)\right) \Phi\left(\mathcal{C}^{\mathrm{I}} ; \mathcal{C}^{\mathrm{II}}\right) \psi^{\mathrm{I}}\left(\mathcal{C}^{\mathrm{I}}\right) d \mathcal{C}^{\mathrm{I}}
\end{aligned}
$$

Henceforth, we will omit explicitly stating the dependencies of the functions wherever obvious. Noting that $\partial \Phi / \partial \mathbf{r}_{i_{\mu}}$ $=-\left(1 / n_{\mu}\right) \partial \Phi / \partial \mathbf{r}_{\mu}$, and multiplying Eq. (1) with $\Phi$ and integrating over all level I configuration space, we obtain the continuity equation for the level II probability field,

$$
\frac{\partial \psi^{\mathrm{II}}}{\partial t}=-\sum_{\mu=1}^{N_{\mathrm{II}}} \frac{\partial}{\partial \mathbf{r}_{\mu}} \cdot\left(\overline{\mathbf{v}}_{\mu} \psi^{\mathrm{II}}\right) .
$$

Next, we sum the force balance Eq. (5) over all $i_{\mu}$ $=1, \ldots, n_{\mu}$, and take the constrained mean of both sides of that equation to obtain the force balance for the $\mu$ th subchain,

$$
\overline{\mathbf{F}}_{\mu}^{h}+\overline{\mathbf{F}}_{\mu}^{B}+\overline{\mathbf{F}}_{\mu}^{U}=\mathbf{0}
$$

where the constrained mean hydrodynamic, Brownian or intramolecular potential forces on the $\mu$ th subchain are given by 


$$
\overline{\mathbf{F}}_{\mu}^{\cdots}=\int\left(\sum_{i_{\mu}=1}^{n_{\mu}} \mathbf{F}_{i_{\mu}}^{\cdots}\right) \psi^{\mathrm{I} \mid \mathrm{II}} d \mathcal{C}^{\mathrm{I} \mid \mathrm{II}}=\frac{1}{\psi^{\mathrm{II}}} \int\left(\sum_{i_{\mu}=1}^{n_{\mu}} \mathbf{F}_{i_{\mu}}^{\cdots}\right) \Phi \psi^{\mathrm{I}} d \mathcal{C}^{\mathrm{I}}
$$

We define here for subsequent use a constrained mean segmental density of the $\mu$ th subchain at some arbitrary location $\mathbf{r}$,

$$
\bar{\rho}_{\mu}\left(\mathbf{r} ; \mathcal{C}^{\mathrm{II}}\right) \equiv \int\left(\frac{1}{n_{\mu}} \sum_{i_{\mu}=1}^{n_{\mu}} \delta\left(\mathbf{r}-\mathbf{r}_{i_{\mu}}\right)\right) \psi^{\mathrm{I} \mid \mathrm{II}} d \mathcal{C}^{\mathrm{I} \mid \mathrm{II}} .
$$

The density field above is normalized in the sense that $\int \bar{\rho}_{\mu}\left(\mathbf{r} ; \mathcal{C}^{\mathrm{II}}\right) d \mathbf{r}=1$. In defining this density field, we begin to picture the polymer molecule as a chain of $N_{\text {II }}$ smoothed density fields, or "blobs," whose centers of masses are located at $\mathbf{r}_{1}, \ldots, \mathbf{r}_{N_{\mathrm{II}}}$. Corresponding to the density field $\bar{\rho}_{\mu}$ above, is a hydrodynamic-force density field,

$$
\overline{\boldsymbol{h}}_{\mu}\left(\mathbf{r} ; \mathcal{C}^{\mathrm{II}}\right) \equiv \int\left(\sum_{i_{\mu}=1}^{n_{\mu}} \delta\left(\mathbf{r}-\mathbf{r}_{i_{\mu}}\right) \mathbf{F}_{i_{\mu}}^{h}\right) \psi^{\mathrm{I}|| \mathrm{I}} d \mathcal{C}^{\mathrm{I} \mid \mathrm{II}},
$$

such that $\int \overline{\boldsymbol{h}}_{\mu}(\mathbf{r}) d \mathbf{r}=\overline{\mathbf{F}}_{\mu}^{h}$. We will also encounter later the two-point correlation function,

$$
\begin{aligned}
\overline{\boldsymbol{j}}_{\mu \nu}\left(\mathbf{r}, \mathbf{r}^{\prime} ; \mathcal{C}^{\mathrm{II}}\right) \equiv & \int\left(\frac{1}{n_{\mu}} \sum_{i_{\mu}=1}^{n_{\mu}} \delta\left(\mathbf{r}-\mathbf{r}_{i_{\mu}}\right)\right) \\
& \times\left(\sum_{j_{\nu}=1}^{n_{\nu}} \delta\left(\mathbf{r}^{\prime}-\mathbf{r}_{j_{\nu}}\right) \mathbf{F}_{j_{\nu}}^{h}\right) \psi^{\mathrm{I}|| \mathrm{II}} d \mathcal{C}^{\mathrm{I} \mid \mathrm{II}},
\end{aligned}
$$

for which $\iint \overline{\boldsymbol{j}}_{\mu \nu} d \mathbf{r} d \mathbf{r}^{\prime}=\overline{\mathbf{F}}_{\nu^{\prime}}^{h}$

We now apply the definitions above to derive expressions for coarse-grained Brownian, intramolecular potential, and hydrodynamic forces.

\section{A. Coarse-graining Brownian and intramolecular forces}

Substituting from Eq. (6) for $\mathbf{F}_{i_{\mu}}^{B}$ in Eq. (16) above, we obtain the constrained-mean Brownian force on the $\mu$ th subchain as

$$
\overline{\mathbf{F}}_{\mu}^{B}=-k_{B} T \frac{\partial}{\partial \mathbf{r}_{\mu}} \ln \psi^{\mathrm{II}}\left(\mathcal{C}^{\mathrm{II}}\right) .
$$

Further, noting that $\mathbf{F}_{i_{\mu}}^{U}\left(\mathcal{C}^{\mathrm{I}}\right)=-\partial U\left(\mathcal{C}^{\mathrm{I}}\right) / \partial \mathbf{r}_{i_{\mu}}$, and that the total intramolecular potential $U\left(\mathcal{C}^{\mathrm{I}}\right)=-k_{B} T \ln \psi_{\mathrm{eq}}^{\mathrm{I}}\left(\mathcal{C}^{\mathrm{I}}\right)$ (modulo an additive constant) where $\psi_{\mathrm{eq}}^{\mathrm{I}}$ is the equilibrium level I distribution, we obtain the constrained-mean intramolecular force on subchain $\mu$ due to the potential $U$,

$$
\begin{aligned}
\overline{\mathbf{F}}_{\mu}^{U} & =k_{B} T \sum_{i_{\mu}=1}^{n_{\mu}} \int \frac{\partial \ln \psi_{\mathrm{eq}}^{\mathrm{I}}}{\partial \mathbf{r}_{i_{\mu}}} \psi^{\mathrm{I} \mid \mathrm{II}} d \mathcal{C}^{\mathrm{I} \mid \mathrm{II}} \\
& =k_{B} T \sum_{i_{\mu}=1}^{n_{\mu}} \frac{1}{\psi^{\mathrm{II}}} \int \frac{\partial \ln \psi_{\mathrm{eq}}^{\mathrm{I}}}{\partial \mathbf{r}_{i_{\mu}}} \Phi \psi^{\mathrm{I}} d \mathcal{C}^{\mathrm{I}} .
\end{aligned}
$$

At equilibrium, the expression above reduces to $\overline{\mathbf{F}}_{\mu \text { eq }}^{U}$ $=k_{B} T \partial \ln \psi_{\mathrm{eq}}^{\mathrm{II}} / \partial \mathbf{r}_{\mu}$.

\section{B. Coarse-graining hydrodynamic drag forces}

Substituting from Eq. (2) for $\mathbf{F}_{i_{\mu}}^{h}$ in the definition for the constrained-mean hydrodynamic force on the $\mu$ th subchain, we obtain

$$
\begin{aligned}
\overline{\mathbf{F}}_{\mu}^{h}= & -n_{\mu} \zeta_{0}\left(\overline{\mathbf{v}}_{\mu}-\boldsymbol{\kappa} \cdot \mathbf{r}_{\mu}\right) \\
& -\zeta_{0} \sum_{i_{\mu}=1}^{n_{\mu}} \sum_{\nu=1}^{N_{\text {II }}} \sum_{j_{\nu}=1}^{n_{\nu}} \int \boldsymbol{\Omega}\left(\mathbf{r}_{i_{\mu}}, \mathbf{r}_{j_{\nu}}\right) \cdot \mathbf{F}_{j_{\nu}}^{h} \psi^{\mathrm{I}|| \mathrm{I}} d \mathcal{C}^{\mathrm{I} \mid \mathrm{II}},
\end{aligned}
$$

which can be combined with the definition of the two-point correlation function in Eq. (19) to yield

$$
\overline{\mathbf{F}}_{\mu}^{h}=-n_{\mu} \zeta_{0}\left(\overline{\mathbf{v}}_{\mu}-\boldsymbol{\kappa} \cdot \mathbf{r}_{\mu}+\sum_{\nu=1}^{N_{\mathrm{II}}} \iint \boldsymbol{\Omega}\left(\mathbf{r}, \mathbf{r}^{\prime}\right) \cdot \overline{\boldsymbol{j}}_{\mu \nu} d \mathbf{r} d \mathbf{r}^{\prime}\right) .
$$

We note here that in the second term on the right-hand side, the summation over all $\nu$ includes $\nu=\mu$. In the absence of HI, we simply have $\overline{\mathbf{F}}_{\mu}^{h}=-n_{\mu} \zeta_{0}\left(\overline{\mathbf{v}}_{\mu}-\boldsymbol{\kappa} \cdot \mathbf{r}_{\mu}\right)$.

Thus far, we have made no approximations. In order to simplify further, we now assume that

$$
\overline{\boldsymbol{j}}_{\mu \nu}\left(\mathbf{r}, \mathbf{r}^{\prime} ; \mathcal{C}^{\mathrm{II}}\right) \approx \overline{\boldsymbol{\rho}}\left(\mathbf{r} ; \mathcal{C}^{\mathrm{II}}\right) \overline{\boldsymbol{h}}_{\nu}\left(\mathbf{r}^{\prime} ; \mathcal{C}^{\mathrm{II}}\right) .
$$

In other words, two-point correlations in the fluctuations in the segmental and hydrodynamic force densities in the blobs are assumed to be negligible. We further assume that the spatial variation of the blob density and hydrodynamic force density fields are similar in the sense that

$$
\overline{\boldsymbol{h}}_{\mu}\left(\mathbf{r} ; \mathcal{C}^{\mathrm{II}}\right)=\overline{\mathbf{F}}_{\mu}^{h}\left(\mathcal{C}^{\mathrm{II}}\right) \bar{\rho}_{\mu}\left(\mathbf{r} ; \mathcal{C}^{\mathrm{II}}\right) .
$$

With these assumptions, the double-integral on the righthand side of Eq. (23) can now be simplified as

$$
\iint \boldsymbol{\Omega}\left(\mathbf{r}, \mathbf{r}^{\prime}\right) \cdot \overline{\boldsymbol{j}}_{\mu \nu} d \mathbf{r} d \mathbf{r}^{\prime}=\overline{\mathbf{\Omega}}_{\mu \nu} \cdot \overline{\mathbf{F}}_{\nu}^{h}
$$

where the tensor $\overline{\mathbf{\Omega}}_{\mu \nu}$ describing HI between the $\mu$ th and $\nu$ th blobs is defined as

$$
\overline{\boldsymbol{\Omega}}_{\mu \nu}\left(\mathcal{C}^{\mathrm{II}}\right) \equiv \iint \bar{\rho}_{\mu}\left(\mathbf{r} ; \mathcal{C}^{\mathrm{II}}\right) \boldsymbol{\Omega}\left(\mathbf{r}, \mathbf{r}^{\prime}\right) \bar{\rho}_{\nu}\left(\mathbf{r}^{\prime} ; \mathcal{C}^{\mathrm{II}}\right) d \mathbf{r} d \mathbf{r}^{\prime} .
$$

Substituting from the equations above in Eq. (23), and rearranging, we obtain

$$
\overline{\mathbf{v}}_{\mu}=\boldsymbol{\kappa} \cdot \mathbf{r}_{\mu}-\sum_{\mu=1}^{N_{\mathrm{II}}} \overline{\mathbf{D}}_{\mu \nu} \cdot \overline{\mathbf{F}}_{\nu}^{h},
$$

where the diffusion tensor,

$$
\overline{\mathbf{D}}_{\mu \nu} \equiv \frac{\delta_{\mu \nu}}{n_{\mu} \zeta_{0}} \boldsymbol{\delta}+\overline{\mathbf{\Omega}}_{\mu \nu}
$$

is the $(\mu, \nu)$ th block of the $3 N_{\mathrm{II}} \times 3 N_{\mathrm{II}}$ mobility matrix for the level II description.

In the complete absence of $\mathrm{HI}$ between all the $N_{\mathrm{I}}$ segments of the level I description, since $\boldsymbol{\Omega}=0$, one would ob- 
tain $\overline{\mathbf{D}}_{\mu \nu}=\delta_{\mu \nu}\left(n_{\mu} \zeta_{0}\right)^{-1} \boldsymbol{\delta}$. From Eq. (27), we see that $\overline{\boldsymbol{\Omega}}_{\mu \nu}$ $=\overline{\boldsymbol{\Omega}}_{\nu \mu}^{\dagger}=\overline{\boldsymbol{\Omega}}_{\nu \mu}$, and therefore the overall level II mobility matrix is symmetric, irrespective of whether or not the chain is partitioned into subchains of equal contour lengths.

Following the steps summarized earlier for deriving the level I FPE, we obtain starting from the level II continuity equation, Eq. (14), using Eq. (28), and the force balance Eq. (15), and substituting from Eq. (20), the level II FPE,

$$
\begin{aligned}
\frac{\partial \psi^{\mathrm{II}}}{\partial t}= & -\sum_{\mu=1}^{N_{\mathrm{II}}} \frac{\partial}{\partial \mathbf{r}_{\mu}} \cdot\left(\boldsymbol{\kappa} \cdot \mathbf{r}_{\mu}+\sum_{\nu=1}^{N_{\mathrm{II}}} \overline{\mathbf{D}}_{\mu \nu} \cdot \overline{\mathbf{F}}_{\nu}^{U}\right) \psi^{\mathrm{II}} \\
& +k_{B} T \sum_{\mu, \nu=1}^{N_{\mathrm{II}}} \frac{\partial}{\partial \mathbf{r}_{\mu}} \cdot \overline{\mathbf{D}}_{\mu \nu} \cdot \frac{\partial \psi^{\mathrm{II}}}{\partial \mathbf{r}_{\mu}}
\end{aligned}
$$

in which the diffusion tensors $\overline{\mathbf{D}}_{\mu \nu}$ are given by Eqs. (29) and (27).

We consider next the effect of coarse graining on the equations for measurable macroscopic observables in the dilute polymer solution.

\section{Macroscopic observables}

Macroscopic observables-such as the mean-squared end-to-end distance of a polymer molecule or the polymer contribution to the total fluid stress tensor-are physical quantities of interest which are obtained as expectations of functions of the configurational variables. For instance, the mean-square end-to-end distance of the polymer in terms of level I variables is

$$
R_{e}^{2}=\left\langle\left(\mathbf{r}_{1, \mathrm{I}}-\mathbf{r}_{N_{\mathrm{I}}}\right)^{2}\right\rangle .
$$

Here, we use $\mathbf{r}_{1, \mathrm{I}}$ to distinguish the level I variable denoting the bead at the chain end from the variable $\mathbf{r}_{1, \mathrm{II}}$ denoting the center of mass of the first blob in the coarser level II description. On coarse graining to level II, we can write the equation above as

$$
R_{e}^{2}=\left\langle\overline{\mathbf{r}_{1, \mathrm{I}}^{2}}-2 \overline{\left(\mathbf{r}_{1, \mathrm{I}} \cdot \mathbf{r}_{N_{\mathrm{I}}}\right)}+\overline{\mathbf{r}_{N_{\mathrm{I}}}^{2}}\right\rangle,
$$

where $\overline{\mathbf{r}_{1, I}^{2}}, \overline{\left(\mathbf{r}_{1, \mathrm{I}} \cdot \mathbf{r}_{N_{\mathrm{I}}}\right)}$, and $\overline{\mathbf{r}_{N_{\mathrm{I}}}}$ are constrained averages and hence are functions of level II variables. The dependence of quantities such as $\overline{\mathbf{r}_{1, \mathrm{I}}^{2}}$ on the level II variables is in general unknown a priori. To overcome this problem, we need to first assume that fluctuations in $\mathbf{r}_{1, \mathrm{I}}$ and $\mathbf{r}_{N_{\mathrm{I}}}$ about their constrained averages, and their cross correlations, are negligible, so that $\overline{\mathbf{r}_{1, \mathrm{I}}^{2}}=\overline{\mathbf{r}_{1, \mathrm{I}}}, \overline{\mathbf{r}_{N_{\mathrm{I}}}^{2}}=\overline{\mathbf{r}_{N_{\mathrm{I}}}}$, and $\overline{\mathbf{r}_{1, \mathrm{I}} \cdot \mathbf{r}_{N_{\mathrm{I}}}}=\overline{\mathbf{r}_{1, \mathrm{I}}} \cdot \overline{\mathbf{r}_{N_{\mathrm{I}}}}$. Thus we obtain for the coarse-grained model,

$$
R_{e}^{2}=\left\langle\left(\overline{\mathbf{r}_{1, \mathrm{I}}}-\overline{\mathbf{r}_{N_{\mathrm{I}}}}\right)^{2}\right\rangle .
$$

The function $\overline{\mathbf{r}_{1, \mathrm{I}}}$, which is the constrained-mean position of the chain end, is clearly different from $\mathbf{r}_{1, \mathrm{II}}$, the center of mass of the first blob. To relate the unknown functions $\overline{\mathbf{r}_{1, \mathrm{I}}}$ and $\overline{\mathbf{r}_{N_{\mathrm{I}}}}$ simply to level II variables, we define a level II connector vector as

$$
\mathbf{Q}_{\mu} \equiv \mathbf{r}_{\mu+1}-\mathbf{r}_{\mu}, \quad \mu=1, \ldots, N_{\mathrm{II}}-1 .
$$

For chains without excluded volume interactions, we esti- mate the constrained mean positions of the ends of the molecule as [7]

$$
\overline{\mathbf{r}_{1, \mathrm{I}}}=\mathbf{r}_{1, \mathrm{II}}-\frac{1}{2} \mathbf{Q}_{1} \quad \text { and } \quad \overline{\mathbf{r}_{N_{\mathrm{I}}}}=\mathbf{r}_{N_{\mathrm{II}}}+\frac{1}{2} \mathbf{Q}_{N_{\mathrm{II}}-1} .
$$

We hence obtain

$$
R_{e}^{2}=\left\langle\left[\left(\mathbf{r}_{1, \mathrm{II}}-\mathbf{r}_{N_{\mathrm{II}}}\right)+\frac{1}{2}\left(\mathbf{Q}_{1}+\mathbf{Q}_{N_{\mathrm{II}}-1}\right)\right]^{2}\right\rangle .
$$

Similarly, starting with the modified Kramers' equation [3] for the polymer contribution to the nonequilibrium extra stress tensor at level I,

$$
\frac{1}{n_{p} k_{B} T} \boldsymbol{\tau}_{p}=-\frac{1}{k_{B} T} \sum_{i=1}^{N_{\mathrm{I}}}\left\langle\left(\mathbf{r}_{i}-\mathbf{r}_{c}\right) \mathbf{F}_{i}^{h}\right\rangle,
$$

where $\mathbf{r}_{c}=\left(1 / N_{\mathrm{I}}\right) \sum_{j=1}^{N_{\mathrm{I}}} \mathbf{r}_{j}$ is the polymer center of mass, we can show that it is necessary to use the assumption postulated in Eq. (24) to obtain the modified Kramers' equation at level II for $\tau_{p}$,

$$
\frac{1}{n_{p} k_{B} T} \boldsymbol{\tau}_{p}=-\frac{1}{k_{B} T} \sum_{\mu=1}^{N_{\mathrm{II}}}\left\langle\left(\mathbf{r}_{\mu}-\mathbf{r}_{c}\right) \overline{\mathbf{F}}_{\mu}^{h}\right\rangle .
$$

In terms of the level II variables, $\mathbf{r}_{c}=\left(1 / N_{\mathrm{I}}\right) \sum_{\mu=1}^{N_{\text {II }}} n_{\mu} \mathbf{r}_{\mu}$. Substituting for $\overline{\mathbf{F}}_{\mu}^{h}$ in the equation above from the force balance in Eq. (15), and using Eq. (20) leads to the Kramers' equation [3],

$$
\frac{1}{n_{p} k_{B} T} \boldsymbol{\tau}_{p}=\frac{1}{k_{B} T} \sum_{\mu=1}^{N_{\mathrm{II}}}\left\langle\left(\mathbf{r}_{\mu}-\mathbf{r}_{c}\right) \overline{\mathbf{F}}_{\mu}^{U}\right\rangle+\left(N_{\mathrm{II}}-1\right) \boldsymbol{\delta} .
$$

In Eq. (39) above, as well as in the level II FPE in Eq. (30), we encounter the functions $\overline{\mathbf{F}}_{\mu}^{U}$ and $\overline{\mathbf{D}}_{\mu \nu}$ which are formally related to the level II configuration through Eq. (21), and Eqs. (27) and (29), respectively. Their computation however requires the knowledge of the level I distribution $\psi^{\mathrm{I}}$, or at least the conditional probability $\psi^{\mid[\mid I I}$, for its evaluation. As a first step in overcoming this hurdle, we consider below an approximation to obtain a more explicit relation connecting the interblob HI tensor in Eq. (27) to the level II configuration variables.

\section{Gaussian blobs}

We define the constrained-mean gyration tensor of the $\mu$ th subchain as

$$
\boldsymbol{\gamma}_{\mu}\left(\boldsymbol{C}^{\mathrm{II}}\right) \equiv \frac{1}{n_{\mu}} \sum_{i_{\mu}=1}^{n_{\mu}} \int\left(\mathbf{r}_{i_{\mu}}-\boldsymbol{c}_{\mu}\right)\left(\mathbf{r}_{i_{\mu}}-\boldsymbol{c}_{\mu}\right) \psi^{\mathrm{I} \mid \mathrm{II}} d \boldsymbol{C}^{\mathrm{I} \mid \mathrm{II}},
$$

where we recall $\mathbf{c}_{\mu}=\left(1 / n_{\mu}\right) \sum_{i_{\mu}=1}^{n_{\mu}} \mathbf{r}_{i_{\mu}}$. Using the definition of the blob density field $\bar{\rho}_{\mu}$ in Eq. (17), we can show that the tensor $\gamma_{\mu}\left(\mathcal{C}^{\mathrm{I}}\right)$ is the second (central) moment of the $\mu$ th blob density field,

$$
\boldsymbol{\gamma}_{\mu}\left(\mathcal{C}^{\mathrm{II}}\right)=\int\left(\mathbf{r}-\mathbf{r}_{\mu}\right)\left(\mathbf{r}-\mathbf{r}_{\mu}\right) \bar{\rho}_{\mu}\left(\mathbf{r} ; \mathcal{C}^{\mathrm{II}}\right) d \mathbf{r} .
$$

In what follows, the tensors $\gamma_{\mu}$ are referred to as the "blob shape" tensors. Let us now assume that we are somehow able 
to calculate exactly $\boldsymbol{\gamma}_{\mu}$ for any blob, given any level II configuration $\mathcal{C}^{\text {II }}$. We now approximate the blob density field $\bar{\rho}_{\mu}$ as a Gaussian distribution with a second moment equal to the exactly calculated $\gamma_{\mu}$. That is, we assume

$$
\bar{\rho}_{\mu}\left(\mathbf{r} ; \mathbf{r}_{\mu}, \boldsymbol{\gamma}_{\mu}\right)=\frac{\exp \left[-(1 / 2)\left(\mathbf{r}-\mathbf{r}_{\mu}\right) \cdot \boldsymbol{\gamma}_{\mu}^{-1} \cdot\left(\mathbf{r}-\mathbf{r}_{\mu}\right)\right]}{\sqrt{(2 \pi)^{3} \operatorname{det}\left[\boldsymbol{\gamma}_{\mu}\right]}} .
$$

For the function $\boldsymbol{\Omega}\left(\mathbf{r}, \mathbf{r}^{\prime}\right)$ in the integrand on the right-hand side of Eq. (27), we use the Oseen-Burgers tensor,

$$
\boldsymbol{\Omega}^{\mathrm{OB}}\left(\mathbf{r}, \mathbf{r}^{\prime}\right)=\frac{1}{8 \pi \eta_{s}|\Delta \mathbf{r}|}\left(\boldsymbol{\delta}+\frac{\Delta \mathbf{r} \Delta \mathbf{r}}{|\Delta \mathbf{r}|^{2}}\right),
$$

with $\Delta \mathbf{r}=\mathbf{r}-\mathbf{r}^{\prime}$. Using a method described by Zylka and Öttinger [8] in a somewhat different context, we obtain after some straightforward algebraic manipulation an expression describing HI between a pair of Gaussian blobs,

$$
\begin{aligned}
\overline{\mathbf{\Omega}}_{\mu \nu}\left(\mathbf{r}_{\nu \mu}, \boldsymbol{\gamma}_{\mu}, \boldsymbol{\gamma}_{\nu}\right)= & \frac{1}{4(2 \pi)^{3 / 2} \eta_{s}} \int_{0}^{\infty}\left[\boldsymbol{\delta}+\left(\boldsymbol{\gamma}_{\mu \nu}+\mathbf{r}_{\nu \mu} \mathbf{r}_{\nu \mu}\right)\right. \\
& \left.\cdot \boldsymbol{A}^{-1}(u)-\boldsymbol{A}^{-1}(u) \cdot\left(\boldsymbol{\gamma}_{\mu \nu} \cdot \mathbf{r}_{\nu \mu} \mathbf{r}_{\nu \mu}\right) \cdot \boldsymbol{A}^{-1}(u)\right] \\
& \times \frac{\exp \left[-\frac{1}{2} \boldsymbol{A}^{-1}(u): \mathbf{r}_{\nu \mu} \mathbf{r}_{\nu \mu}\right]}{\sqrt{\operatorname{det} \boldsymbol{A}(u)}} d u,
\end{aligned}
$$

where $\mathbf{r}_{\nu \mu}=\mathbf{r}_{\nu}-\mathbf{r}_{\mu}$ is the displacement between the centers of the $\nu$ th and $\mu$ th blobs, $\gamma_{\mu \nu}=\gamma_{\mu}+\gamma_{\nu}$, and $\boldsymbol{A}(u)=\gamma_{\mu \nu}+u \boldsymbol{\delta}$. We will henceforth refer to $\overline{\boldsymbol{\Omega}}_{\mu \nu}$ calculated using the equation above as the Gaussian-blob HI (GB-HI) tensor.

We summarize below some salient features of the GB-HI tensor. First, Eq. (44) shows that the interblob HI tensor depends on $\mathbf{r}_{\nu \mu}$ the separation of two blobs, as well as the sizes and orientations of the blobs, which enter the description through the shape tensors, $\gamma_{\mu}$ and $\gamma_{\nu}$. Second, the GB-HI tensor satisfies the symmetry relations, $\overline{\mathbf{\Omega}}_{\mu \nu}=\overline{\mathbf{\Omega}}_{\mu \nu}^{\dagger}$, and $\overline{\mathbf{\Omega}}_{\mu \nu}=\overline{\boldsymbol{\Omega}}_{\nu \mu}$. Further, it can be shown that the function $\overline{\boldsymbol{\Omega}}_{\mu \nu}\left(\mathbf{r}_{\nu \mu}, \boldsymbol{\gamma}_{\mu}, \boldsymbol{\gamma}_{\nu}\right)$ is homogeneous, with

$$
\overline{\boldsymbol{\Omega}}_{\mu \nu}\left(\lambda \mathbf{r}_{\nu \mu}, \lambda^{2} \boldsymbol{\gamma}_{\mu}, \lambda^{2} \boldsymbol{\gamma}_{\nu}\right)=\lambda^{-1} \overline{\boldsymbol{\Omega}}_{\mu \nu}\left(\mathbf{r}_{\nu \mu}, \boldsymbol{\gamma}_{\mu}, \boldsymbol{\gamma}_{\nu}\right) .
$$

This property has its origin in the $1 / r$ decay of the OseenBurgers tensor.

It is useful to consider the case where both the $\mu$ th and $\nu$ th blobs are isotropic, and hence $\gamma_{\mu \nu}=\gamma_{\mu \nu} \delta$. In this case, we obtain from Eq. (44),

$$
\begin{aligned}
\overline{\mathbf{\Omega}}_{\mu \nu}= & \frac{1}{8 \sqrt{2} \pi^{3 / 2} \eta_{s} \gamma_{\mu \nu}^{1 / 2}}\left[\left(\frac{\Gamma(1 / 2, \theta)}{\theta^{1 / 2}}+\frac{\Gamma(3 / 2, \theta)}{\theta^{3 / 2}}\right) \boldsymbol{\delta}\right. \\
& \left.+2 \theta\left(\frac{\Gamma(3 / 2, \theta)}{\theta^{3 / 2}}-\frac{\Gamma(5 / 2, \theta)}{\theta^{5 / 2}}\right) \frac{\mathbf{r}_{\nu \mu} \mathbf{r}_{\nu \mu}}{r_{\nu \mu}^{2}}\right],
\end{aligned}
$$

where $\theta=r_{\nu \mu}^{2} / 2 \gamma_{\mu \nu}$, and

$$
\Gamma(a, x)=\int_{0}^{x} t^{a-1} e^{-t} d t,
$$

is the incomplete gamma function. As the distance between the blobs becomes very large compared to their dimensions, and $\quad \theta \rightarrow \infty, \quad \Gamma(1 / 2, \theta) \rightarrow \Gamma(1 / 2, \infty)=\sqrt{\pi}, \quad$ and $\quad \Gamma(3 / 2, \theta)$ $\rightarrow \Gamma(3 / 2, \infty)=\sqrt{\pi} / 2$. Thus, in the limit of large separations, we see that interblob GB-HI tensor approaches the OseenBurgers tensor.

We also need to consider what happens when the distance between a pair of blobs $\mathbf{r}_{\nu \mu}=\mathbf{0}$. In this case, Eq. (44) reduces to

$$
\overline{\boldsymbol{\Omega}}_{\mu \nu}=\frac{1}{4(2 \pi)^{3 / 2} \eta_{s}} \int_{0}^{\infty}\left[\boldsymbol{\delta}+\boldsymbol{\gamma}_{\mu \nu} \cdot \boldsymbol{A}^{-1}(u)\right] \frac{1}{\sqrt{\operatorname{det} \boldsymbol{A}(u)}} d u .
$$

Zylka [9] showed that the integral on the right-hand side can be efficiently evaluated using standard routines for elliptic integrals. This result further shows that the GB-HI tensor remains well behaved even when blobs fully overlap. It is well known that if the Oseen-Burgers tensor were to be used to model $\mathrm{HI}$ between spherical particles, the $1 / r$ singularity in the Oseen-Burgers tensor will result in the overall mobility matrix of the system becoming nonpositive definite for those configurations where the distance between any pair of particles becomes much smaller than their radii. However, the singularity of $1 / r$ at $r=0$ poses no problems when it appears as $\int(1 / r) d \mathbf{r}$ in an integration in three dimensions. This fact has been exploited previously in preaveraged treatments of HI such as the Zimm [10], consistent averaging [11], and Gaussian approximations [12], all of which use Gaussian averages of the Oseen-Burgers tensor. Similarly, the GB-HI tensor also eliminates the effect of the singularity in the Oseen-Burgers tensor, and the overall level II mobility matrix consequently remains positive definite even when blobs overlap. The Gaussian-blob HI is thus similar in this respect to the Rotne-Prager-Yamakawa HI tensor $[13,14]$ which is commonly used in simulations of bead-spring chain models of dilute polymer solutions to ensure positive definiteness of the mobility matrix for all chain configurations.

Equation (48) above is necessary for the calculation of the diffusion tensors $\overline{\mathbf{D}}_{\mu \mu}$, which are in fact the mobility tensors of the individual Gaussian blobs. In the complete absence of any HI between the beads of the level I representation of the polymer chain, the "bare" mobility of the $\mu$ th blob is $\overline{\mathbf{D}}_{\mu \mu}$ $=\left(n \zeta_{0}\right)^{-1} \boldsymbol{\delta}$. The inclusion of HI between different segments of the same subchain leads to "dressed" blob mobilities $\overline{\mathbf{D}}_{\mu \mu}=\left(n_{\mu} \zeta_{0}\right)^{-1} \boldsymbol{\delta}+\overline{\mathbf{\Omega}}_{\mu \mu}$. The tensor $\overline{\mathbf{\Omega}}_{\mu \mu}$ accounts for the contribution of intrablob HI to the mobility of the blob, and is obtained by substituting $2 \gamma_{\mu}$ in place of $\gamma_{\mu \nu}$ in Eq. (48) above, and

$$
\overline{\boldsymbol{\Omega}}_{\mu \mu}=\frac{1}{4(2 \pi)^{3 / 2} \eta_{s}} \int_{0}^{\infty}\left[\boldsymbol{\delta}+2 \boldsymbol{\gamma}_{\mu} \cdot \boldsymbol{A}^{-1}(u)\right] \frac{1}{\sqrt{\operatorname{det} \boldsymbol{A}(u)}} d u,
$$

where $\boldsymbol{A}(u)=2 \boldsymbol{\gamma}_{\mu}+u \boldsymbol{\delta}$. Thus, $\overline{\boldsymbol{\Omega}}_{\mu \mu}$ is a function of the shape tensor $\gamma_{\mu}$ alone. If the shape tensors are axisymmetric, and $\gamma_{\mu}=\gamma_{\mu}^{\perp}\left(\boldsymbol{\delta}-\mathbf{u}_{\mu} \mathbf{u}_{\mu}\right)+\gamma_{\mu}^{\|} \mathbf{u}_{\mu} \mathbf{u}_{\mu}$, where $\mathbf{u}_{\mu}$ is a unit vector specifying the orientation of the principal axis of the $\mu$ th blob, $\overline{\boldsymbol{\Omega}}_{\mu \mu}$ is also of the form $\bar{\Omega}_{\mu}^{\perp}\left(\boldsymbol{\delta}-\mathbf{u}_{\mu} \mathbf{u}_{\mu}\right)+\bar{\Omega}_{\mu}^{\|} \mathbf{u}_{\mu} \mathbf{u}_{\mu}$. In this case, 
the integral in Eq. (49) above can be reduced analytically [7]. If a blob is spherically isotropic, and $\gamma_{\mu}=\gamma_{\mu} \boldsymbol{\delta}$, we obtain from Eq. (49),

$$
\overline{\mathbf{\Omega}}_{\mu \mu}=\frac{\sqrt{1 / \pi}}{6 \pi \eta_{s} \gamma_{\mu}^{1 / 2}} \boldsymbol{\delta} .
$$

We note here that as $N_{\mathrm{II}} \rightarrow N_{\mathrm{I}}$, and $n_{\mu} \rightarrow 1, \gamma_{\mu}$ must approach $\left(3 a_{0}^{2} / 5\right) \boldsymbol{\delta}$ (the second central moment of a spherically uniform particle of radius $a_{0}$ ), and in principle the mobilities of the individual blobs should approach $\zeta_{0}^{-1} \boldsymbol{\delta}$. However, setting $n_{\mu}=1$, and $\gamma_{\mu}=3 a_{0}^{2} / 5$ in Eq. (50) instead leads to $\overline{\mathbf{D}}_{\mu \mu}$ $=\zeta_{0}^{-1}[1+\sqrt{5 /(3 \pi)}] \boldsymbol{\delta}$. The additional $\sqrt{5 /(3 \pi)} \boldsymbol{\delta}$ term arises because, in deriving Eq. (44) starting from Eq. (27), we did not explicitly prevent level I beads from overlapping. In addition, by using the Oseen-Burgers tensor to simplify the integral in Eq. (44), we have neglected details of short-range interactions between segments at the level I description of the polymer chain. Although one could use a more accurate description of $\mathrm{HI}$ at level I instead of the Oseen-Burgers tensor, we anticipate that the additional detail will only lead to correction terms to the already complicated expression for the GB-HI tensor in Eq. (44). The use of the simple OseenBurgers tensor is justified as long as one is interested in using the coarse-grained model to explore universal features of the dynamics of dilute polymer solutions.

\section{E. Local equilibrium ansatz}

The assumption of a Gaussian-blob density has enabled us to obtain an explicit functional form for the HI tensor $\overline{\mathbf{\Omega}}_{\mu \nu}$, which depends on $\mathbf{r}_{\nu \mu}=\mathbf{r}_{\nu}-\mathbf{r}_{\mu}$ and the shape tensors $\gamma_{\mu}$ and $\gamma_{\nu}$. In order to perform computations, we need to know the dependence of the shape tensors $\boldsymbol{\gamma}_{\mu}$ on the level II configuration. An exact calculation using the definition in Eq. (41) with the true (non-Gaussian, in general) blob density requires knowledge of the unknown instantaneous conditional probability $\psi^{\mathrm{I} \mid \mathrm{II}}$. We face a similar problem with respect to the coarse-grained intramolecular force $\overline{\mathbf{F}}_{\mu}^{U}$, as mentioned earlier. To break free from these constraints, we take recourse to the following local equilibrium ansatz:

$$
\psi^{\mathrm{I} \mid \mathrm{II}}\left(\mathcal{C}^{\mathrm{I}} \mid \mathcal{C}^{\mathrm{II}}\right) \approx \psi_{\mathrm{eq}}^{\mathrm{I} \mid \mathrm{II}}\left(\mathcal{C}^{\mathrm{I}} \mid \mathcal{C}^{\mathrm{II}}\right) .
$$

This is a reasonable assumption when the characteristic relaxation time scales of the slower level II variables and the faster level I variables are widely separated, and the solvent flow field is weak enough so that the individual level I subchain configurations are always equilibrated with the constraints specified by $\mathcal{C}^{\text {II }}$.

Substituting the local equilibrium ansatz above in the definition of $\overline{\mathbf{F}}_{\mu}^{U}$ in Eq. (21), we directly obtain for the coarse-grained intermolecular potential force,

$$
\overline{\mathbf{F}}_{\mu}^{U}\left(\mathcal{C}^{\mathrm{II}}\right)=\overline{\mathbf{F}}_{\mu, \mathrm{eq}}^{U}\left(\mathcal{C}^{\mathrm{II}}\right)=k_{B} T \frac{\partial \ln \psi_{\mathrm{eq}}^{\mathrm{II}}}{\partial \mathbf{r}_{\mu}} .
$$

This expression now permits us to calculate the functional dependence of $\overline{\mathbf{F}}_{\mu}^{U}$ on $\mathcal{C}^{\mathrm{II}}$ variables using equilibrium statis- tical mechanics, since the equilibrium distribution $\psi_{\mathrm{eq}}^{\mathrm{II}}$ can be in principle calculated exactly according to

$$
\psi_{\mathrm{eq}}^{\mathrm{II}}\left(\mathcal{C}^{\mathrm{II}}\right)=\frac{\int \Phi e^{-U / k_{B} T} d \mathcal{C}^{\mathrm{I}}}{\int e^{-U / k_{B} T} d \mathcal{C}^{\mathrm{I}}} .
$$

Since we can also-again, in principle-calculate the conditional probability distribution $\psi_{\text {eq }}^{\text {III }}$, we can use the gyration tensors obtained from

$$
\boldsymbol{\gamma}_{\mu}\left(\mathcal{C}^{\mathrm{II}}\right)=\frac{1}{n_{\mu}} \sum_{i_{\mu}=1}^{n_{\mu}} \int\left(\mathbf{r}_{i_{\mu}}-\boldsymbol{c}_{\mu}\right)\left(\mathbf{r}_{i_{\mu}}-\boldsymbol{c}_{\mu}\right) \psi_{\mathrm{eq}}^{\mathrm{I} \mid \mathrm{II}} d \mathcal{C}^{\mathrm{I} \mid \mathrm{II}}
$$

as the shape tensors in Eq. (27) for evaluating the GB-HI tensors.

The local equilibrium ansatz can be made irrespective of whether or not excluded volume interactions are included. For highly flexible chains with no excluded volume or other nonlocal interactions, subchains are statistically uncorrelated at equilibrium in the sense that $\psi_{\mathrm{eq}}^{\mathrm{II}}$ and $\psi_{\mathrm{eq}}^{\mid \mathrm{II}}$ are both factorizable as

$$
\psi_{\mathrm{eq}}^{\mathrm{II}}\left(\mathcal{C}^{\mathrm{II}}\right)=\prod_{\mu=1}^{N_{\mathrm{II}}} \psi_{\mu, \mathrm{eq}}^{\mathrm{II}}\left(\mathcal{C}^{\mathrm{II}}\right)
$$

and

$$
\psi_{\mathrm{eq}}^{\mathrm{I} \mid \mathrm{II}}\left(\mathcal{C}^{\mathrm{I}} \mid \mathcal{C}^{\mathrm{II}}\right)=\prod_{\mu=1}^{N_{\mathrm{II}}} \psi_{\mu, \mathrm{eq}}^{\mathrm{I} \mid \mathrm{II}}\left(\mathcal{C}_{\mu}^{\mathrm{I}} \mid \mathcal{C}^{\mathrm{II}}\right),
$$

where $\mathcal{C}_{\mu}^{\mathrm{I}}$ is the subset of $\mathcal{C}^{\mathrm{I}}$ containing the position vectors of the beads of the $\mu$ th subchain alone. In this special case, further simplifications can be anticipated. For example, the shape tensor for the $\mu$-blob $\gamma_{\mu}$ is independent of the orientations and dimensions of the other blobs in the chain.

With the assumption of local equilibrium, both the coarsegrained intramolecular potential forces $\overline{\mathbf{F}}_{\mu}$ and the blobshape tensors $\gamma_{\mu}$ can be calculated for any given level II configuration $\mathcal{C}^{\mathrm{Il}}$ without requiring any further knowledge of either the level I configuration variables or the instantaneous nonequilibrium conditional probability distribution $\psi^{|| I I}$. Once the tensors $\gamma_{\mu}$ are known, the GB-HI tensors $\overline{\mathbf{\Omega}}_{\mu \nu}$ can be calculated using Eq. (44), and all the diffusion tensors $\overline{\mathbf{D}}_{\mu \nu}$ can next be obtained, substituting in the level II FPE, Eq. (30).

Unfortunately, detailed expressions even in the simple noexcluded-volume case are unavailable for the equilibrium distributions $\psi_{\mathrm{eq}}^{\mathrm{II}}$ and $\psi_{\mathrm{eq}}^{\mathrm{I} \mid \mathrm{II}}$, especially when the centers of masses of subchains are constrained to fixed locations in space. For the preliminary simulations in this study, we have used approximate expressions for $\overline{\mathbf{F}}_{\mu}^{U}$ and $\gamma_{\mu}$ which are discussed in the following sections.

\section{Entropic spring force}

If we ignore excluded volume and other such nonlocal interactions, and the total level I intramolecular potential ac- 
counts for those interactions along the polymer backbone that are responsible for maintaining its connectivity, flexibility and total length, we assume that the mean intramolecular force on the $\mu$ th blob $\overline{\mathbf{F}}_{\mu}^{U}$ is of the form

$$
\overline{\mathbf{F}}_{\mu}^{U}= \begin{cases}\overline{\mathbf{F}}_{1}^{c} & \text { if } \mu=1, \\ \overline{\mathbf{F}}_{\mu}^{c}-\overline{\mathbf{F}}_{\mu-1}^{c} & \text { if } \mu=2, \ldots, N_{\mathrm{II}}-1, \\ -\overline{\mathbf{F}}_{N_{\mathrm{II}}-1}^{c} & \text { if } \mu=N_{\mathrm{II}},\end{cases}
$$

where $\overline{\mathbf{F}}_{\mu}^{c}$ defined for $\mu=1, \ldots, N_{\text {II }}-1$ is a "connector" force that depends solely on the corresponding connector vector $\mathbf{Q}_{\mu}$. We further assume that the connector force function $\overline{\mathbf{F}}_{\mu}^{c}\left(\mathbf{Q}_{\mu}\right)$ has a form identical to the mean entropic resistance exerted at equilibrium when the subchain end-to-end vector is held fixed at $\mathbf{Q}_{\mu}$. Recently, Underhill and Doyle [15] have derived force laws relating $\overline{\mathbf{F}}_{\mu}^{c}$ to $\mathbf{Q}_{\mu}$ for coarse-grained descriptions of semiflexible wormlike chains. In this study, we however restrict our attention to simulations of flexible subchains $\left(L_{\mu} \gg b_{K}\right)$, in which case the entropic resistance is given by the inverse Langevin force law $[3,16]$. Since the inverse Langevin function is difficult to implement in simulations, we use the following "finitely extensible nonlinear elastic" (FENE) force law which is widely regarded as a good approximation to the inverse Langevin expression $[3,17]$,

$$
\overline{\mathbf{F}}_{\mu}^{c}=\frac{H_{\mu}}{1-Q_{\mu}^{2} / L_{\mu}^{2}} \mathbf{Q}_{\mu} .
$$

The constants $H_{\mu}$ are phenomenological spring constants, whose values are fixed typically by requiring that the equilibrium mean-squared end-to-end distance predicted with the FENE force at the level II description match $R_{e, \text { eq }}^{2}=b_{K}^{2} N_{K}$ at the level I description [7].

\section{Blob-shape tensors}

We assume that in the absence of excluded volume interactions, individual blobs are axisymmetric, and the shape tensor $\gamma_{\mu}$ is solely dependent on the constrained-mean endto-end vector of the $\mu$ th subchain, which is estimated to be (Fig. 2; [7])

$$
\mathbf{T}_{\mu}= \begin{cases}\mathbf{Q}_{1} & \text { if } \mu=1, \\ \frac{1}{2}\left(\mathbf{Q}_{\mu}+\mathbf{Q}_{\mu-1}\right) & \text { if } 2 \leqslant \mu \leqslant N_{\mathrm{II}}-1, \\ \mathbf{Q}_{N_{\mathrm{II}}-1} & \text { if } \mu=N_{\mathrm{II}} .\end{cases}
$$

We will refer to the magnitude $T_{\mu}=\left|\mathbf{T}_{\mu}\right|$ as the blob stretch, and to the unit vector $\mathbf{u}_{\mu}=\left(1 / T_{\mu}\right) \mathbf{T}_{\mu}$ as the blob orientation vector. It may be noted that with this definition of $\mathbf{T}_{\mu}$, the Gaussian-blob model does not consist of touching axisymmetric ellipsoids in general. Given a subchain contour length, $L_{\mu}$, the axisymmetric shape tensor is

$$
\boldsymbol{\gamma}_{\mu}=\gamma_{\mu}^{\perp}\left(\boldsymbol{\delta}-\mathbf{u}_{\mu} \mathbf{u}_{\mu}\right)+\gamma_{\mu}^{\|} \mathbf{u}_{\mu} \mathbf{u}_{\mu},
$$

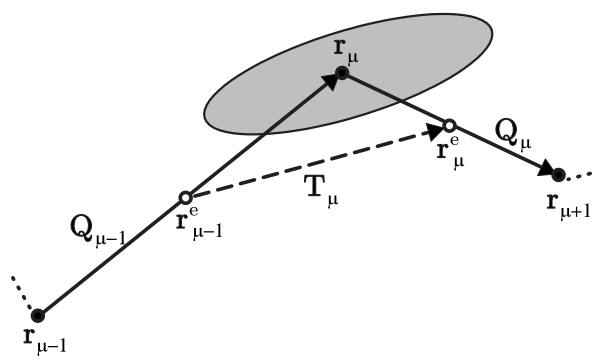

FIG. 2. Schematic showing the relationship of the blob shape.

where the longitudinal and transverse eigenvalues of $\gamma_{\mu}, \gamma_{\mu}^{\|}$, and $\gamma_{\mu}^{\perp}$ are functions of the blob stretch $T_{\mu}$. As in the case of the entropic spring force, we further assume that the dependence of $\gamma_{\mu}^{\perp}$ and $\gamma_{\mu}^{\|}$on the blob stretch $T_{\mu}$ is the same as that of the gyration tensor eigenvalues of a subchain whose ends are held apart by a distance $T_{\mu}$ at equilibrium.

To the best of our knowledge, there are no general analytical results available describing the complete dependence of the constrained equilibrium gyration tensor on the end-toend stretch, even for flexible chains. We have therefore obtained the functions $\gamma_{\mu}^{\|}\left(T_{\mu}\right)$ and $\gamma_{\mu}^{\perp}\left(T_{\mu}\right)$ for a wide range of $N_{K / S}=N_{K} N_{\text {II }}$ values using BD simulations [7]. The shape tensors thus obtained serve as inputs for the calculation of the GB-HI tensors $\boldsymbol{\Omega}_{\mu \nu}$ and the diffusion tensors $\overline{\mathbf{D}}_{\mu \nu}$.

In short, the local equilibrium ansatz allows us to calculate the forces $\overline{\mathbf{F}}_{\mu}^{U}$ and the diffusion tensors $\overline{\mathbf{D}}_{\mu \nu}$ for any given level II configuration $\mathcal{C}^{\mathrm{II}}=\left\{\mathbf{r}_{1}, \ldots, \mathbf{r}_{N_{\mathrm{II}}}\right\}$ of the dissolved polymer chain. These functions of the configuration can be substituted in the level II FPE to obtain the time evolution of the probability distribution $\psi^{\text {II }}$. In addition, the coarse-graining procedure described above introduces no new undetermined constants. The only parameters in the coarse-grained model are the following: $T$ and $\eta_{s}$ are properties of the solvent continuum, $L, N_{K}$, and $a_{0}$ are parameters that characterize the continuous-chain model of the dissolved polymer, and $N_{\text {II }}$ and the set $\left\{L_{\mu} \mid \mu=1, \ldots, N_{\mathrm{II}}\right\}$ of partitions of the chain contour define the coarse graining of the polymer chain. The time evolution of macroscopic observables such as the polymer stress tensor $\boldsymbol{\tau}_{p}$ can be obtained by the technique of BD simulations which is described shortly.

\section{F. Conventional bead-spring model}

We close this discussion of coarse-graining of intramolecular $\mathrm{HI}$ in dilute polymer solutions with a summary of the commonly used bead-spring chain model with Rotne-PragerYamakawa (RPY) HI. The conventional bead-spring model is also a level II coarse-grained representation of the polymer molecule, typically into equal-sized segments. The hydrodynamic characteristics of a segment are lumped into a single "bead" friction coefficient, $\zeta^{\mathrm{BSM}}=6 \pi a^{\mathrm{BSM}} \eta_{s}$, where $a^{\mathrm{BSM}}$ 
isthe bead radius. Thus, in the conventional model, spherical beads of fixed radii take the place of the Gaussian blobs proposed in this study. The beads are further connected by entropic FENE springs. Using RPY-HI between the beads leads to a Fokker-Planck equation for this bead-spring model that has the same form as Eq. (30), but with the following diffusion tensors in place of $\overline{\mathbf{D}}_{\mu \nu}$ :

$$
\mathbf{D}_{\mu \nu}^{\mathrm{BSM}}=\frac{\delta_{\mu \nu}}{\zeta^{\mathrm{BSM}}} \boldsymbol{\delta}+\boldsymbol{\Omega}_{\mu \nu}^{\mathrm{RPY}},
$$

where

$$
\boldsymbol{\Omega}_{\mu \mu}^{\mathrm{RPY}}=\mathbf{0},
$$

and, when $\mu \neq \nu$,

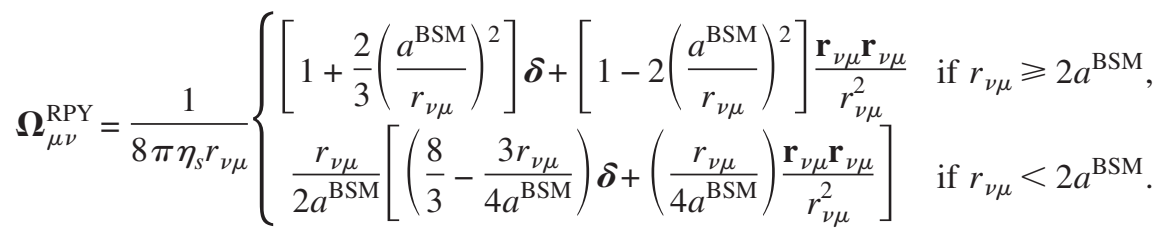

The bead radius $a^{\mathrm{BSM}}$ has no direct connection with the level I parameter $a_{0}$ which no longer appears in the equations. The parameter $a^{\mathrm{BSM}}$ is thus a free parameter whose value must be specified as an input. It is customary to do this in terms of the dimensionless hydrodynamic interaction parameter defined as

$$
h^{*} \equiv \sqrt{\frac{3}{\pi}} \frac{a^{\mathrm{BSM}}}{\left\langle Q^{2}\right\rangle_{\mathrm{eq}}^{1 / 2}},
$$

where $\left\langle Q^{2}\right\rangle_{\mathrm{eq}}$ is the equilibrium mean-square connector length in the absence of excluded volume interactions. From studies on the universal scaling behavior of theta solutions, it is known that a choice of $h^{*} \approx 0.25$ is particularly effective in reproducing the near-equilibrium dynamics of long molecules even with bead-spring chains with small $[\sim O(10)]$ values of $N_{\text {II }}$ [18]. In situations where molecules experience stretching and are highly anisotropic, one must either choose a different value of $h^{*}$ in order to accurately describe the behavior of long molecules with a low value of $N_{\text {II }}$ [5], or choose larger values of $N_{\text {II }}$ while retaining $h^{*} \approx 0.25$ [4].

For the level I representation of the polymer molecule as a chain of touching beads, $\left\langle Q^{2}\right\rangle_{\mathrm{eq}}=L / N_{\mathrm{I}}=2 a_{0}$, and hence $h^{*}$ $=\sqrt{(3 / \pi)}(1 / 2)=0.49$ for that representation. Therefore, with a choice of $h^{*}=0.49$, one expects that simulation results obtained with coarser bead-spring representations should converge as $N_{\mathrm{II}} \rightarrow N_{\mathrm{I}}$ to results that would have been obtained with a level I structural representation but with RPY-HI between the touching beads. If, on the other hand, one keeps $h^{*}$ fixed at 0.25 , simulation results converge as $N_{\mathrm{II}} \rightarrow N_{\mathrm{I}}$ to those corresponding to a model consisting of $N_{\mathrm{I}}$ nontouching beads. Those features of the results that remain sensitive to the value of $h^{*}$ chosen even as $N_{\mathrm{II}} \rightarrow N_{\mathrm{I}} \gg 1$ can then be said to depend strongly on the local details in the modeling of HI.

We now turn to a summary of the simulation algorithms used in this study.

\section{BROWNIAN DYNAMICS SIMULATIONS}

In BD simulations, an ensemble of stochastic trajectories is generated by several independent integrations of the fol- lowing set of $N_{\text {II }}$ coupled Itô stochastic differential equations (SDE's) that is equivalent to the FPE in Eq. (30) [19],

$$
\begin{aligned}
d \mathbf{r}_{\mu}= & \left(\boldsymbol{\kappa} \cdot \mathbf{r}_{\mu}+\sum_{\nu=1}^{N_{\text {II }}} \overline{\mathbf{D}}_{\mu \nu} \cdot \overline{\mathbf{F}}_{\nu}^{U}+k_{B} T \sum_{\nu=1}^{N_{\text {II }}} \frac{\partial}{\partial \mathbf{r}_{\nu}} \cdot \overline{\mathbf{D}}_{\mu \nu}\right) d t \\
& +\sqrt{2 k_{B} T} \sum_{\nu=1}^{N_{\text {II }}} \overline{\boldsymbol{B}}_{\mu \nu} \cdot d \boldsymbol{W}_{\nu} .
\end{aligned}
$$

Starting with a known initial level II configuration $\mathcal{C}^{\text {II }}$ $=\left\{\mathbf{r}_{1}, \ldots, \mathbf{r}_{N_{\mathrm{II}}}\right\}$ at $t=0$, the set of SDE's represented by the equation above can be integrated forward in time, and a stochastic trajectory can be generated. In Eq. (62) above, $\boldsymbol{W}_{\nu}$ is a vector each of whose three components is an independent Wiener process. The forces $\overline{\mathbf{F}}_{\mu}^{U}$ are calculated using Eqs. (57) and (56). To calculate the diffusion tensors $\overline{\mathbf{D}}_{\mu \nu}$, we first obtain the blob orientation vectors, $\mathbf{u}_{\mu}$ and blob stretches, $T_{\mu}$, using Eq. (58). The transverse and longitudinal components of the shape tensors, $\gamma_{\mu}$ are then calculated using the procedure described in Sec. II E 2 above. The blob mobility tensors $\overline{\mathbf{D}}_{\mu \mu}$ are next obtained using Eq. (29) with $\mu=\nu$, and the expressions for the transverse and longitudinal eigenvalues of the tensors $\overline{\mathbf{\Omega}}_{\mu \mu}$ [7]. For $\mu \neq \nu$, the diffusion tensors $\overline{\mathbf{D}}_{\mu \nu}$ require evaluation of the integral in Eq. (44), which is performed numerically in our simulations. In the absence of $\mathrm{HI}$, $\overline{\mathbf{D}}_{\mu \nu}=\left(n_{\mu} \zeta_{0}\right)^{-1} \delta_{\mu \nu} \boldsymbol{\delta}$. The dimensionless matrices $\overline{\boldsymbol{B}}_{\mu \nu}$ are chosen such that they satisfy the fluctuation-dissipation theorem, $\sum_{\theta=1}^{N_{I I}} \overline{\boldsymbol{B}}_{\mu \theta} \cdot \overline{\boldsymbol{B}}_{\theta \nu}^{\dagger}=\overline{\mathbf{D}}_{\mu \nu}$. The Chebyshev polynomial approximation method is used to obtain the diffusion term $\Delta \boldsymbol{S}_{\mu}$ $=\Sigma_{\nu=1}^{N_{\text {II }}} \overline{\boldsymbol{B}}_{\mu \nu} \cdot \Delta \boldsymbol{W}_{\nu}[18,20,21]$, where $\Delta \boldsymbol{W}_{\nu}$ are the Wiener increments corresponding to a time-step size of $\Delta t$.

Although the Oseen-Burgers tensor itself is divergence free, the contribution to the drift term [in large parentheses in Eq. (62) above] stemming from the divergence

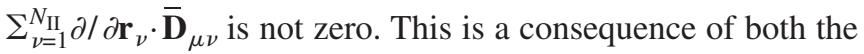
anisotropy and configuration dependence of the blob-shape tensors. It can be shown that if the blob shapes are forced to 
be spherical with constant radii, the divergence term vanishes for the GB-HI model as well. In contrast, the divergence term is zero in the conventional model with RPY-HI, which greatly simplifies computation with that model. However, this simplification comes at the expense of the inability of the model to account for the effect of subchain anisotropy on HI. As soon as the assumption of spherical beads is relaxed, and/or if the bead radius becomes configuration dependent, the divergence term is nonzero, as observed even in a one-dimensional dumbbell model with a variable drag coefficient [22].

Since the direct implementation of the analytical calculation of the divergence term is tedious, we use the following numerical estimate. If $\mathbf{R}$ is the $3 N_{\mathrm{II}}$-dimensional vector composed of the components of all the position vectors $\mathbf{r}_{\mu}$ at some time $t$, and $\Delta \mathbf{W}$ is the $3 N_{\mathrm{II}^{-}}$-dimensional vector composed of the components of the Wiener increments $\Delta \boldsymbol{W}_{\mu}$, then it can be shown that

$$
\frac{1}{2}\langle[\overline{\mathbf{D}}(\mathbf{R}+\Delta \mathbf{W})-\overline{\mathbf{D}}(\mathbf{R}-\Delta \mathbf{W})] \cdot \Delta \mathbf{W}\rangle=\left\langle\nabla_{\mathbf{R}} \cdot \overline{\mathbf{D}}\right\rangle \Delta t+O\left(\Delta t^{2}\right) .
$$

In other words, the distribution of stochastic trajectories obtained after integration following Euler time-discrete SDE,

$$
\begin{aligned}
\Delta \mathbf{r}_{\mu}= & \left(\boldsymbol{\kappa} \cdot \mathbf{r}_{\mu}+\sum_{\nu=1}^{N_{\mathrm{II}}} \overline{\mathbf{D}}_{\mu \nu}(\mathbf{R}) \cdot \overline{\mathbf{F}}_{\mu}^{U}\right) \Delta t+\sqrt{2 k_{B} T} \sum_{\nu=1}^{N_{\mathrm{II}}} \overline{\mathbf{B}}_{\mu \nu} \cdot \Delta \mathbf{W}_{\nu} \\
& +\frac{k_{B} T}{2} \sum_{\nu=1}^{N_{\mathrm{II}}}\left[\overline{\mathbf{D}}_{\mu \nu}(\mathbf{R}+\Delta \mathbf{W})-\overline{\mathbf{D}}_{\mu \nu}(\mathbf{R}-\Delta \mathbf{W})\right] \cdot \Delta \mathbf{W}_{\nu},
\end{aligned}
$$

converges in the limit of zero time-step size to the distribution that would have been obtained by integrating the SDE in Eq. (62). We use Eq. (64) in a predictor step, followed by a corrector step in which the spring forces are treated implicitly. This semi-implicit predictor-corrector integration scheme has been described in detail elsewhere $[5,23,24]$.

\section{RESULTS AND DISCUSSION}

In an ideal coarse-graining scheme, for a given fixed value of $N_{\mathrm{I}}$, the time evolutions of macroscopic observables obtained after solving the level II FPE would be independent of the choice of $N_{\mathrm{II}}<N_{\mathrm{I}}$, and thus be identical to those obtained by solving the level I FPE. The scheme developed in this study however uses a number of approximations, as a result of which simulation results obtained for any fixed value of $N_{\mathrm{I}}$ can be expected to vary with $N_{\mathrm{II}}$. We present here a preliminary assessment of the efficacy of the Gaussian-blob coarse-graining scheme, by comparing the $N_{\text {II }}$ variation in its predictions with that observed with conventional coarse graining as a bead-spring chain with RPY-HI between spherical beads of fixed radii. The comparison is made for the relaxation of the mean-squared end-to-end distance of a molecule $R_{e}^{2}$, and the polymer contribution to the first normal-stress difference $N_{1, p}=\left|\tau_{p, x x}-\tau_{p, y y}\right|$ in simulations where the initial ensemble consists of chains all stretched out in the $x$ direction to $90 \%$ their full extension. Chains subse-

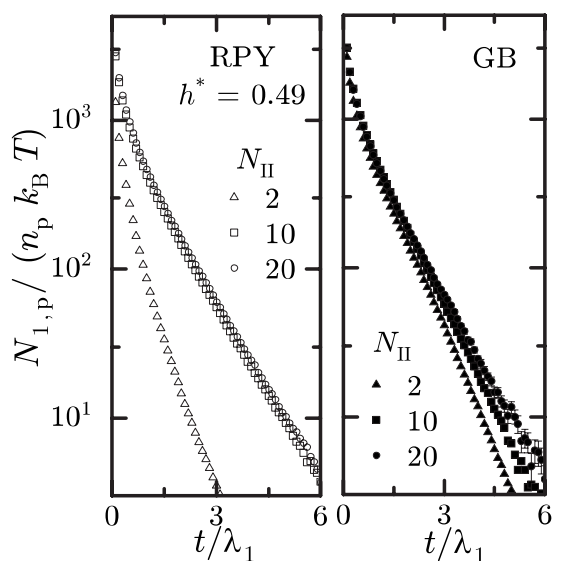

FIG. 3. Relaxation of the first-normal stress difference in an ensemble of chains all stretched initially to $90 \%$ full extension in the $x$ direction.

quently relax under quiescent $(\boldsymbol{\kappa}=\mathbf{0})$ conditions.

For the sake of simplicity, we choose $a_{0}=b_{k} / 2$, which means that $N_{\mathrm{I}}=L /\left(2 a_{0}\right)=N_{K}$. Further, we use equal-sized partitions in our coarse graining, and hence $n_{\mu}=n=N_{\mathrm{I}} / N_{\text {II }}$ $=N_{K} / N_{\mathrm{II}}=N_{K / S}$, where $N_{K / S}$ denotes the number of Kuhn segments per coarse-grained subchain. As mentioned earlier, the calculation of the spring-constant $H$ is shown in Ref. [7]. The remaining free parameters, $T, \eta_{s}$, and $b_{K}$, can be removed from the equations by appropriately rescaling all quantities using a thermal energy scale $\epsilon=k_{B} T$, a subchain length scale $\ell_{S}=b_{K}^{2} N_{K / S} / 3$, and a subchain diffusive time scale $\lambda_{S}$ $=n \zeta_{0} \ell_{S}^{2} /\left(4 k_{B} T\right)$. For the simulations in this study, we have used $N_{K}=500$, and values of $N_{\text {II }}$ ranging from 2 to 50 for the conventional model, and 2 to 20 for the Gaussian-blob model.

Results are presented below in Figs. 3 and 4 in terms of the dimensionless ratios, $R_{e}^{2} / b_{K}^{2}$ and $N_{1, p} /\left(n_{p} k_{B} T\right)$. The time axis in the figures is in terms of the ratio $t / \lambda_{1}$, where

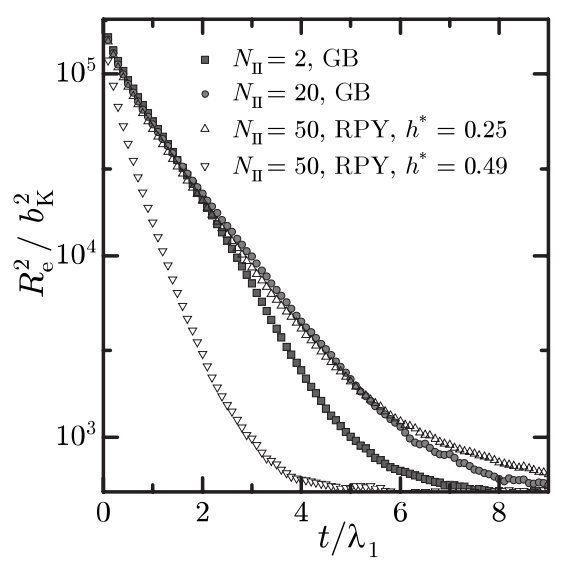

FIG. 4. Relaxation of the mean-squared end-to-end distance in an ensemble of chains all stretched initially to $90 \%$ full extension in the $x$ direction. 


$$
\lambda_{1}=0.21 \frac{\eta_{s} b_{k}^{3} N_{K}^{3 / 2}}{k_{B} T},
$$

is an empirical estimate of the largest near-equilibrium relaxation time of the dissolved polymer molecule obtained in recent simulations [4].

Figure 3 compares the dependence of the results for $N_{1, p} /\left(n_{p} k_{B} T\right)$ on $N_{\text {II }}$ in the Gaussian-blob and conventional bead-spring models. When the whole range of $N_{\text {II }}$, starting from $N_{\text {II }}=2$ (dumbbells) to $N_{\text {II }}=20$ is considered, it is apparent that the results of the Gaussian-blob model show a smaller degree of variation with respect to $N_{\mathrm{II}}$. This is particularly true during the initial relaxation of the stress, when chains are still highly anisotropic. However, the results of the conventional model appear to have converged with respect to $N_{\text {II }}$ by a value of $N_{\text {II }}=10$, whereas the difference between the data obtained for $N_{\mathrm{II}}=10$ and $N_{\mathrm{II}}=20$ with the Gaussian-blob model becomes significant towards the later stages of the relaxation. The reason for this is currently not clearly understood.

It is worthwhile to ask whether the results obtained with the Gaussian-blob model converge to the same values as those of the conventional model with RPY-HI, $N_{\mathrm{II}} \rightarrow N_{\mathrm{I}}$. Figure 4 compares the results of the two models for the relaxation of $R_{e}^{2}$.

For the conventional bead-spring model, we show results for $N_{\mathrm{II}}=50$, for two different values of $h^{*}$. As we may recall from the discussion in Sec. II F, $h^{*}=0.49$ is the choice that, as $N_{\mathrm{II}} \rightarrow N_{\mathrm{I}}$, leads to results obtained with a level I touching bead chain and RPY-HI. Figure 4 also shows results obtained with the conventional model with $h^{*}=0.25$. The large difference in Fig. 4 between the results for the two different $h^{*}$ values indicates that the relaxation of $R_{e}^{2}$ and $N_{1, p}$ for a chain with $N_{K}=500$ can depend considerably on local hydrodynamic detail. Hence, we cannot expect too close an agreement between the results of the two different models even with large values of $N_{\text {II }}$ since the models differ in their handling of near-field HI.

It must be recognized that for any given choice of $N_{\mathrm{II}}$, simulations with GB-HI are necessarily more computationally expensive than those with RPY-HI due to the additional contribution to the drift arising from the non-divergence-free nature of the mobility matrix. Moreover, the expressions for the HI tensor in the RPY model [Eq. (60)] are undoubtedly much easier to implement in computer code than Eq. (44) for interblob and intrablob $\mathrm{HI}$ in the Gaussian-blob model, which requires in addition the prior (albeit one-time) computation of the dependence of the shape tensors on the chain configuration. These difficulties are the price that one may be willing to pay in return for a more refined treatment of HI, if the $N_{\mathrm{II}}$ convergence of model independent features of results is achieved with lesser overall computational expense in the new model than in the conventional model. A clear answer to this question of overall efficiency is not possible with the preliminary results presented in Figs. 3 and 4 which are clearly model dependent (as evidenced by the strong $h^{*}$ dependence of the RPY-HI predictions), and requires a more detailed investigation which is beyond the scope of the present work. Nevertheless, the qualitative agreement between the two models, coupled with the smaller variation of the results of the Gaussian-blob model with respect to $N_{\text {II }}$ observed in Figs. 3 and 4 suggest that the Gaussian-blob model could provide a useful alternative for coarse-graining $\mathrm{HI}$ in polymer solutions.

\section{SUMMARY AND CONCLUSIONS}

In this study, we have developed a systematic framework for coarse-graining hydrodynamic interactions in an isolated polymer molecule in solution. The Gaussian-blob model accounts for the effect of the variation in the instantaneous shapes of the subchains in the coarse-grained representation, on the hydrodynamic interaction within and between subchains. Preliminary results obtained with BD simulations indicate that the Gaussian-blob model achieves a significant reduction in the dependence of results on the degree of coarse graining, when compared to the conventional beadspring model with Rotne-Prager-Yamakawa hydrodynamic interaction.

\section{ACKNOWLEDGMENTS}

This work has been supported through a monetary grant from the Australian Research Council (Discovery Project No. DP0450458), and a CPU-time grant from the Australian Partnership for Advanced Computing. The authors thank Ravi Jagadeeshan, Monash University, for his useful comments and suggestions.
[1] P. Español, Lect. Notes Phys. 640, 69 (2004).

[2] P. Español and F. Vázquez, Philos. Trans. R. Soc. London, Ser. A 360, 383 (2002).

[3] R. B. Bird, C. F. Curtiss, R. C. Armstrong, and O. Hassager, Dynamics of Polymeric Liquids-Volume 2: Kinetic Theory, 2nd ed. (Wiley, New York, 1987).

[4] R. Prabhakar, J. R. Prakash, and T. Sridhar, J. Rheol. 48, 1251 (2004).

[5] C.-C. Hsieh, L. Li, and R. G. Larson, J. Non-Newtonian Fluid Mech. 113, 147 (2003).
[6] C.-C. Hsieh and R. G. Larson, J. Rheol. 48, 995 (2004).

[7] See EPAPS Document No. E-PLEEE8-76-025707 for details on the calculation of orientations, and shape and mobility tensors of blobs in the Gaussian-blob model. The document also contains details on the calculation of the FENE spring constant. For more information on EPAPS, see http://www.aip.org/ pubservs/epaps.html.

[8] W. Zylka and H. C. Öttinger, Macromolecules 24, 484 (1991).

[9] W. Zylka, J. Chem. Phys. 94, 4628 (1991).

[10] B. H. Zimm, J. Chem. Phys. 24, 269 (1956). 
[11] H. C. Öttinger, J. Chem. Phys. 86, 3731 (1987).

[12] H. C. Öttinger, J. Chem. Phys. 90, 463 (1989).

[13] J. Rotne and S. Prager, J. Chem. Phys. 50, 4831 (1969).

[14] H. Yamakawa, J. Chem. Phys. 53, 436 (1970).

[15] P. T. Underhill and P. S. Doyle, J. Rheol. 50, 513 (2006).

[16] H. Yamakawa, Modern Theory of Polymer Solutions (Harper and Row, New York, 1971).

[17] H. R. Warner, Ind. Eng. Chem. Fundam. 11, 379 (1972).

[18] M. Kröger, A. Alba-Pérez, M. Laso, and H. C. Öttinger, J. Chem. Phys. 113, 4767 (2000).

[19] H. C. Öttinger, Stochastic Processes in Polymeric Fluids
(Springer-Verlag, New York, 1996).

[20] M. Fixman, Macromolecules 19, 1204 (1986).

[21] R. M. Jendrejack, M. D. Graham, and J. J. de Pablo, J. Chem. Phys. 113, 2894 (2000).

[22] C. M. Schroeder, E. S. G. Shaqfeh, and S. Chu, Macromolecules 37, 9242 (2004).

[23] R. Prabhakar, Ph.D. thesis, Monash University, 2005 (unpublished).

[24] R. Prabhakar and J. R. Prakash, J. Non-Newtonian Fluid Mech. 116, 163 (2004). 